\title{
Upregulation of lonotropic Glutamate Receptor Subunits within Specific Mesocorticolimbic Regions during Chronic Nicotine Self-Administration
}

\author{
Fan Wang', Hao Chen', Jeffery D Steketee' and Burt M Sharp*,' \\ 'Department of Pharmacology, University of Tennessee Health Science Center, Memphis, TN, USA
}

\begin{abstract}
Nicotine, an addictive substance, is the major psychoactive component in cigarette smoke. Both $\boldsymbol{\alpha}$-amino-3-hydroxy-5-methyl-4isoxazole propionic acid and N-methyl-D-aspartate (NMDA) receptors are essential for the acute stimulative effects of nicotine on the mesocorticolimbic dopamine system, yet little is known about the effects of chronic nicotine treatment on glutamate receptors. Therefore, we used a model of chronic nicotine self-administration (SA), which emulates important aspects of nicotine intake by humans, to determine whether glutamate receptor subunits were affected. After 18 days of saline vs nicotine SA, ionotropic glutamate receptor subunit levels were determined in brain regions within the mesocorticolimbic system by Western blotting. In prefrontal cortex (PFC), the levels of NMDA receptor subunit 2A (NR2A) and NR2B were increased by $67 \%(p=0.04)$ and $83 \%(p=0.027)$, respectively. In the ventral tegmental area (VTA), glutamate receptor subunit $2 / 3(\mathrm{GluR} 2 / 3)$ increased by $34 \%(p=0.0 \mathrm{I}$ I). Nicotine SA did not affect the expression of these subunits in dorsal striatum and nucleus accumbens. These findings suggest that chronic nicotine SA selectively increased the levels of ionotropic glutamate receptor subunits in a brain region-specific manner.

Neuropsychopharmacology (2007) 32, I03-109. doi:I0. I038/sj.npp. I 30 I033; published online I February 2006
\end{abstract}

Keywords: nicotine; ventral tegmental area; prefrontal cortex; NMDA receptor; AMPA receptor; mesocorticolimbic

\section{INTRODUCTION}

The mesocorticolimbic system, which is involved in cognition, stress, and goal-directed motor activity, as well as addiction, involves the ventral tegmental area (VTA), nucleus accumbens (NAcc), prefrontal cortex (PFC), dorsal striatum (ST), hippocampus, and amygdala (Le Moal and Simon, 1991). Nicotine, the major psychoactive component of cigarette smoke, has been shown to stimulate mesocorticolimbic dopamine pathways, which originate in the VTA, resulting in dopamine (DA) secretion within the NAcc and PFC (Corrigall et al, 1992). In doing so, nicotine activates and desensitizes various subtypes of nicotinic cholinergic receptors (nAChRs) located on dopaminergic neurons, GABAergic neurons, and glutamatergic terminals within the VTA, and on dopamine terminals in the projection regions (eg NAcc) (Corrigall et al, 1992). This nicotinic stimulation of dopamine secretion is essential for the reinforcing effects of nicotine (Corrigall et al, 1992).

\footnotetext{
*Correspondence: Dr BM Sharp, Department of Pharmacology, University of Tennessee Health Science Center, Suite 115 Crowe Building, 874 Union Avenue, Memphis, TN 38163 USA, Tel: + I 901 448 6000, Fax: + I 901448 7206, E-mail: bsharp@utmem.edu Received 4 May 2005; revised 4 November 2005; accepted 19 December 2005

Online publication: 28 December 2005 at http://www.acnp.org/ citations/Npp | 22805050292/default.pdf
}

However, other neurotransmitter pathways, such as glutamatergic neurons projecting from PFC to VTA, also are involved in the motivational effects of nicotine (Watkins et al, 2000; Picciotto and Corrigall, 2002).

Synaptic glutamate binds to $N$-methyl-D-aspartate (NMDA) and $\alpha$-amino-3-hydroxy-5-methyl-4-isoxazole propionic acid (AMPA) receptors (ie NR and GluR, respectively), opening postsynaptic cation channels that mediate fast excitatory neurotransmission (Nakanishi et al, 1998). Recent reports have shown that nicotine stimulated VTA glutamate release through presynaptic nicotinic acetylcholine receptors, which in turn excited VTA dopaminergic neurons (Fu et al, 2000; Mansvelder and McGehee, 2000). Nicotine induced glutamate release through $\alpha 7$ subunit-containing nAChRs on glutamate terminals in a VTA slice preparation (Mansvelder and McGehee, 2000). Ultrastructural and in vivo microdialysis studies support the idea that the excitation of VTA dopamine neurons is primarily dependent on glutamatergic efferents from PFC (Sesack and Pickel, 1992; Taber et al, 1995). Using in vivo microdialysis, our lab also showed that nicotine dosedependently stimulated DA release in NAcc, an effect that was abolished by infusing NMDA antagonists (AP-5 or CGS 19755) into VTA (Fu et al, 2000). These findings demonstrate the pivotal role played by glutamatergic pathways in the excitation of mesocorticolimbic dopaminergic neurons by nicotine. 
Nicotine, like other drugs of abuse, elevated the ratio of AMPA/NMDA excitatory postsynaptic currents (EPSC) in midbrain dopamine neurons, consistent with enhanced glutamatergic neurotransmission which contributes to greater dopamine neuron excitability (Saal et al, 2003). Nicotine also enhanced NMDA and/or AMPA-induced synaptosomal release of norepinephrine from the hippocampus and dopamine from the striatum (Risso et al, 2004). These functional studies suggest that nicotine may upregulate ionotropic glutamate receptors, but do not provide insight into the effects of nicotine on the actual expression of ionotropic glutamate receptors in specific brain regions. The expression of GluR1 mRNA in VTA has been found to be increased after acute injection of nicotine directly into VTA (Ferrari et al, 2002). However, the effects of chronic nicotine self-administration (SA) on ionotropic glutamate receptor protein levels have not, to our knowledge, been studied. The aim of this study was to determine whether chronic nicotine SA modulates protein levels of ionotropic glutamate receptor subtypes within mesocorticolimbic regions. To accomplish this, Western blotting with subunit-specific antibodies was used to obtain semiquantitative comparisons within the VTA, PFC, Nacc, and ST.

\section{MATERIALS AND METHODS}

\section{Materials}

(-)Nicotine hydrogen tartrate (all doses calculated as free base) was purchased from Sigma (St Louis, MO). Operant chambers, circuit boards, interface modules, and SA software were from Coulbourn Instruments (Allentown, PA).

\section{Nicotine SA}

SA was performed according to our previously published protocol with minor modifications (Valentine et al, 1997). Initially, all rats used in both control and treatment groups were treated identically. At 7 days after acclimation to a reverse light cycle and handling, male Lewis rats (Harlan Sprague-Dawley, Indianapolis, IN), weighing 250-350 g, received jugular cannulae under xylazine-ketamine anesthesia (13 and $87 \mathrm{mg} / \mathrm{kg}$, i.m., body weight, respectively; Parke-Davis, Morris Plains, NJ) and then were immediately placed into individual operant chambers where they resided for the duration of the study. Rats were allowed to recover for 3 days, during which time they received hourly injections of heparin $(100-200 \mathrm{U} / \mathrm{ml}$ in $50 \mu \mathrm{l})$ and a daily injection of the antibiotic Baytril $(7.6 \mathrm{mg} / \mathrm{kg}$ in $0.1 \mathrm{ml}$, i.v.; Bayer Corp., Shawnee Mission, KS). Then, all rats were randomly assigned to two treatment groups (ie saline or nicotine SA) and jugular lines were filled with either $0.03 \mathrm{mg} / \mathrm{kg}$ nicotine in $200 \mathrm{U} / \mathrm{ml}$ heparinized saline $(50 \mu \mathrm{l}$ delivered over $0.81 \mathrm{~s}$ per $300 \mathrm{gm}$ body weight) or heparinized saline alone. The nicotine solution was freshly prepared every 7-14 days and stored in a foil-wrapped glass bottle at $4^{\circ} \mathrm{C}$. Every day, the nicotine solution remaining in the syringe was discarded and the syringe was refilled.

Each sound-attenuating environmental enclosure was equipped with its own ventilation fan and house light; the light was scheduled to turn off daily at exactly 1030 hours and on again at 2230 hours. The interior operant chamber contained two levers positioned $5 \mathrm{~cm}$ above the floor and a green cue light $1 \mathrm{~cm}$ above each that signaled the availability of nicotine. One lever was randomly assigned as the active bar and would signal the computer-driven pump to deliver a $50 \mu \mathrm{l} / 0.81 \mathrm{~s}$ bolus injection of nicotine or saline when pressed by the rat; pressing the other bar had no consequence. Injections were followed by a $7 \mathrm{~s}$ time-out, during which the green light above the active bar was not illuminated and nicotine or saline were unavailable. Rats were not shaped, conditioned, or food deprived in preparation for lever pressing and were allowed to acquire SA behavior throughout the $23 \mathrm{~h}$.

Environmental enclosures, operant chambers, and syringe pumps were controlled by Coulbourn Habitest Universal LabLink interfaces and computers located in an adjacent room. Each morning, during the final hour of the lights-on cycle (ie at 0930 hours), the clock for the computer program (Coulbourn L2T2) had to be manually reset, necessitating a short interruption in nicotine availability. To accommodate this, as well as to accomplish animal husbandry needs, measurements of body weight, and data downloading, all of the environmental enclosures were opened and the green cue lights were turned off to signal this interruption to the rats. Levers were not retracted, and lever press activity was not recorded. Exactly $1 \mathrm{~h}$ later (1030 hours), house lights were switched off, cue lights were illuminated, nicotine (or saline) was again available, and enclosure doors were shut. All procedures were conducted in accordance with the NIH Guidelines Concerning the Care and Use of Laboratory Animals and were approved by the Animal Care and Use Committee of the University of Tennessee.

Rats were housed in SA chambers for 18 days. By day 8, stable nicotine SA had been achieved in that active lever presses were two-fold greater than inactive lever presses in each animal. All animals continued to self-administer nicotine until day 18.

\section{Brain Punches and Tissue Preparation}

On day 19 , after rats had access to nicotine SA for $4 \mathrm{~h}$, they were anesthetized with isoflurane and decapitated. The average time interval between the last active lever press for nicotine and removal of the animals from operant chambers was $31.8 \mathrm{~min}$; thus, rats were untethered from their chambers $3 \mathrm{~h}$ and $28.2 \pm 18.6 \mathrm{~min}$ (mean \pm SD) after the start of the session. Rat brains were removed and stored at $-80^{\circ} \mathrm{C}$. Frozen brain sections of $0.7 \mathrm{~mm}$ thickness were obtained using arrays of 20 double-edge razor blades (Professional Medical Products Inc. Greenwood, SC), designed and constructed in our laboratory. The blade arrays were assembled using two screws with matching wing-nuts. One $0.7 \mathrm{~mm}$ stainless steel washer per screw (Small Parts, Inc. Miami Lakes, FL) was used to separate each blade. Brains were first equilibrated at $-20^{\circ} \mathrm{C}$ for $10 \mathrm{~min}$. The ventral surface of the brain was then placed on top of the blade array and was gently and briefly pressed downward. Care was taken to ensure that the rostral-caudal axis of the brain was perpendicular to the blades (for coronal sections). The brain and the blades were then 
turned upside down and set on top of a cold $\left(-10^{\circ} \mathrm{C}\right)$ metal plate (FTS systems, Stone Ridge, NY). Using a slight rocking motion, a metal block was applied to gradually press the entire brain into the blades, insuring that the brain was completely embedded within the blades. The assembly then was disassembled on powdered dry ice, and individual brain slices were laid flat on a cold $\left(-10^{\circ} \mathrm{C}\right)$ surface. Brain areas of interest were identified with a dissecting microscope, according to the atlas of Paxinos and Watson (1986), and punched out using a 20 gauge $(0.86 \mathrm{~mm}$ diameter $)$ syringe adapter. Excised tissue was immediately transferred to tubes embedded in dry ice and stored at $-80^{\circ} \mathrm{C}$ until further processing. Dissected tissues were sonicated in $2 \%$ sodium dodecyl sulfate (SDS) solution for $30 \mathrm{~s}$ in an ice water bath. Sonicated tissues were centrifuged at $800 \times g, 5 \mathrm{~min}$. Protein concentrations were measured using the bicinchoninic acid assay (Pierce, Rockford, IL) with bovine serum albumin (BSA) as the standard.

\section{Western Blotting}

To determine ionotropic glutamate receptor expression level differences after treatment, samples of the same brain region from both treatment groups (nicotine $v s$ saline) were loaded in alternating sequence on the same gel.

Samples were diluted in Laemmli buffer (Bio-Rad, Hercules, CA) at a ratio of $1: 2$, and were boiled for $5 \mathrm{~min}$, loaded, and then separated on $10 \%$ polyacrylamide gels in a buffer containing $0.1 \%$ SDS, $192 \mathrm{mM}$ glycine, and $25 \mathrm{mM}$ Tris, $\mathrm{pH}$ 8.3. Proteins were then transferred to polyvinylidene difluoride membranes (Bio-Rad, Hercules, CA) in a buffer containing $192 \mathrm{mM}$ glycine, $20 \%$ methanol, and $25 \mathrm{mM}$ Tris, $\mathrm{pH} 8.3$ at $4^{\circ} \mathrm{C}$ overnight. After transfer, membranes were washed in Tris-buffered saline with Tween-20 (TBST; $100 \mathrm{mM}$ Tris and $0.9 \%$ sodium chloride with $0.1 \%$ Tween 20). Membranes were blocked with agitation by $5 \%$ nonfat dried milk for $2 \mathrm{~h}$ at room temperature (RT). Based on the molecular weight differences between NMDA receptor subunits, AMPA receptor subunits, and $\beta$-actin, blots were carefully cut into three separate sections by visualizing the protein molecular weight markers: upper blots were used to detect NMDA receptor subunits, middle blots to detect AMPA receptor subunits, and lower blots to detect $\beta$-actin in order to evaluate protein loading. Each section contained the samples from all the nicotine-saline pairs studied in these experiments. These blots were incubated with selective antibodies against $\beta$-actin ( $1: 1000$; Sigma, St Louis, MO), glutamate receptor subunit 1 (GluR1; $1: 1000$; Upstate, Lake Placid, NY), GluR2/3 (1:1000; Upstate, Lake Placid, NY), NMDA receptor subunit 1 (NR1;1:400; Santa Cruz, CA), NR2A ( $1: 200$; Santa Cruz, CA), and NR2B (1:500; Upstate, Lake Placid, NY). Blots were then washed in TBST and incubated in horseradish peroxidase-conjugated secondary antibodies ( $1: 3000$ for $\beta$-actin, $1: 1500$ for detecting the others). All antibodies were diluted in the same blocking solution and incubated for $2 \mathrm{~h}$ RT or overnight at $4{ }^{\circ} \mathrm{C}$. Target proteins were visualized by Supersignal (Pierce, Rockford, IL) followed by imaging using a Bio-Rad ChemiDoc instrument with quantitation by Quantity One software (Bio-Rad, Hercules, CA).

\section{Data Analysis}

To determine the specificity of nicotine SA, the number of active $v s$ inactive lever presses per day were compared within each treatment group (ie nicotine and saline). Data are presented as mean $\pm \mathrm{SEM}$. Comparisons of active $v s$ inactive lever presses within treatment groups were made by two-way analysis of variance, using SPSS statistical software, version 12.0 (SPSS Inc., Chicago, IL).

Each sample from a rat was run once on each of the two gels. Optical density values for each lane were normalized to $\beta$-actin to control for variation in loading and transfer. For each sample, normalized values that had been averaged between the two blots were used to conduct statistical comparisons (independent samples $t$-test) to identify treatment effects within a region for each subunit. The mean values of nicotine treatment groups were expressed as a percentage of saline \pm SEM. Treatment differences were considered significant at $p<0.05$.

\section{RESULTS}

\section{Nicotine SA}

The numbers of daily active $v s$ inactive lever presses (mean \pm SEM) are presented in Figure 1. In the nicotine treatment group, the mean level of active lever presses by rats receiving a programmed injection of i.v. nicotine immediately after each active lever press was approximately twice the inactive presses. In this group, active lever presses were significantly higher than inactive $\left(\mathrm{F}_{1,10}=51.49\right.$, $p<0.001)$, whereas no difference was observed in the saline group $\left(\mathrm{F}_{1,10}=3.68, p>0.05\right)$. A comparison of active lever presses in the nicotine $v s$ saline groups also showed a significant treatment effect $\left(F_{1,10}=9.57, p=0.002\right)$.

\section{Chronic Nicotine SA-Induced Upregulation of NMDA and AMPA Receptor Subtypes within Mesocorticolimbic Regions}

In all immunoblots, only one band was visualized. Representative gels from each group are shown in Figures 2-5. NMDA receptor NR2A and NR2B subunits were visualized at a molecular weight of $180 \mathrm{kDa}$. Chronic nicotine SA significantly increased NR2A and NR2B subunit expression levels in PFC by 67\% $(p=0.04)$ and $83 \%$ $(p=0.027)$, respectively (Figure 2$)$. The GluR2/3 subunit was visualized at $110 \mathrm{kDa}$. In VTA, chronic nicotine SA preferentially upregulated the GluR2/3 receptor expression level by $34 \%(p=0.011$; Figure 3$)$. In contrast, Figures 4 and 5 show that NAcc and striatal NMDA and AMPA receptor subunit levels were unaffected by chronic nicotine SA.

The relatively large variance in NR2A levels in NAcc (Figure 4) and the statistical tendency toward a difference between treatment groups $(p=0.06)$ prompted a second experiment. NR2A was unaffected by nicotine SA (immunoblot optical density, expressed as a percentage of the saline group: $100 \pm 12.6$ vs $109.6 \pm 27.5 \%$ (values are mean \pm SEM) for saline vs nicotine, respectively; $p>0.05, n=6$ per treatment group). For comparison, NR1 determinations were also repeated. As previously reported, no treatment effects were found ( $100 \pm 5.5 v s 99.8 \pm 21.1 \%$ for saline vs nicotine, respectively; $p>0.05, n=6$ per treatment group). 


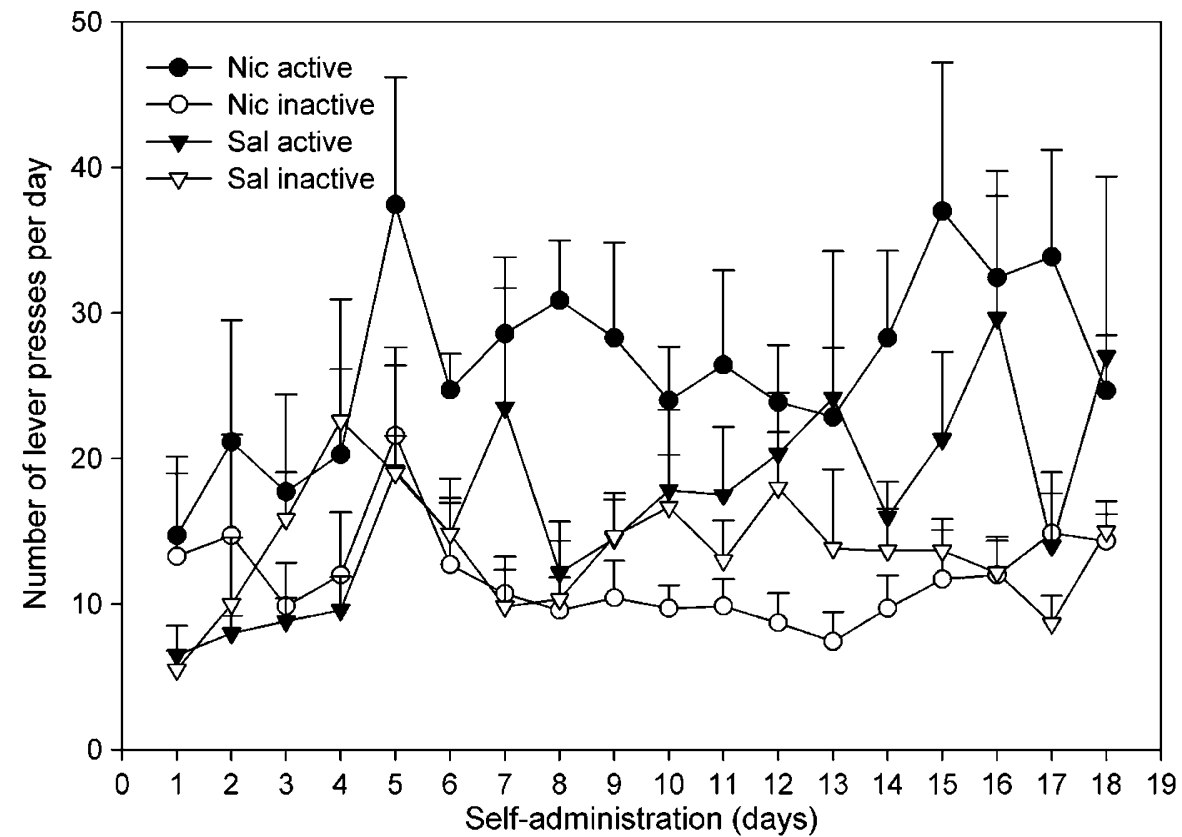

Figure I Lever-pressing activity during I 8 days of nicotine (Nic) SA compared to saline (Sal). The mean numbers ( \pm SEM) of lever presses (active or inactive) per day for the four data sets were analyzed by two-way analysis of variance. In the nicotine group, the difference between active vs inactive lever presses was significant $(F=51.49, p<0.001 ; n=7)$, but not in the saline group $(F=3.68, p>0.05 ; n=6)$. Brain tissues from these animals were used in the immunoblots shown in Figures 2-5.

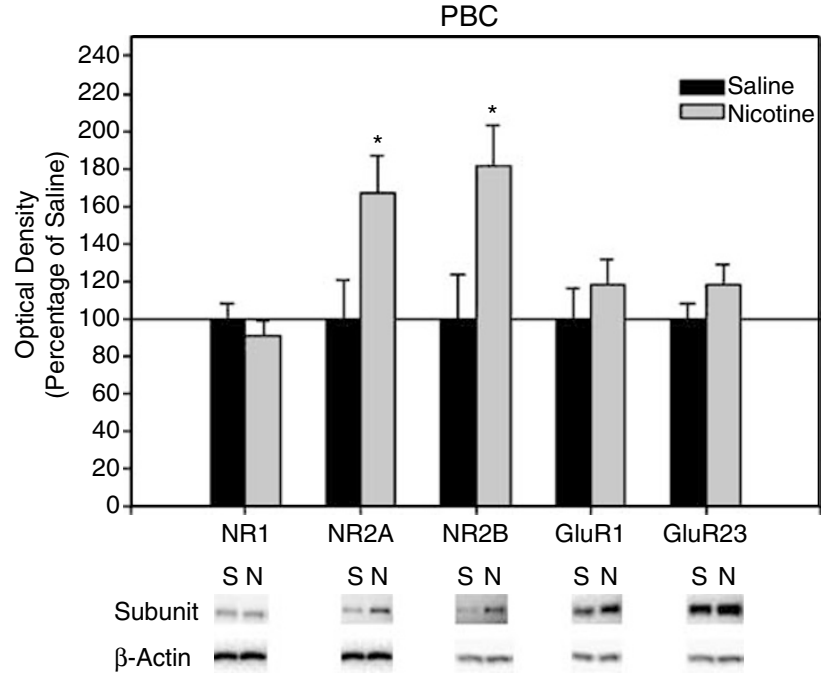

Figure 2 Nicotine SA ( 8 days) upregulated NR2A and NR2B subunit levels within the prefrontal cortex (PFC) of rat brain. Nicotine induced significant increases (67 and $83 \%$ ) in NR2A and NR2B subunit immunoreactivities within the PFC. Data were normalized to $\beta$-actin levels and converted to mean percent of saline \pm SEM for each subunit (nicotine $n=7$, saline $n=6$ ). Representative blots comparing saline (S) vs nicotine $(\mathrm{N})$-treated animals are shown for each subunit. *Significantly different from saline; $p<0.05$, unpaired $t$-test.

\section{DISCUSSION}

In rats chronically self-administering nicotine, several glutamate receptor subunits were upregulated within the mesocorticolimbic pathway. These include NR2A and NR2B receptor subunits within PFC and GluR2/3 subunits within

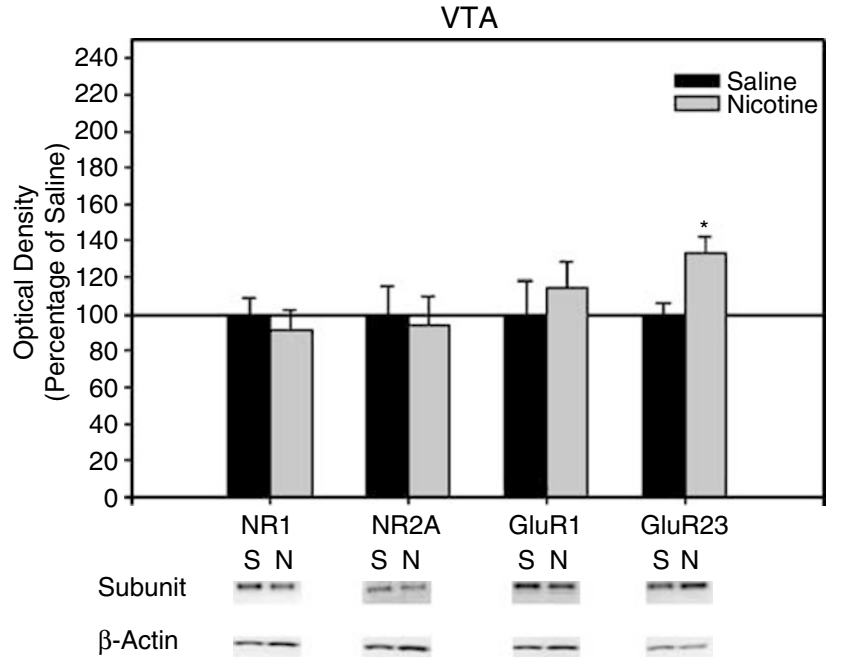

Figure 3 Nicotine SA (18 days) increased GluR2/3 subunit immunoreactivity within the ventral tegmental area (VTA) of rat brain. Nicotine increased GluR2/3 subunit levels in this region by $34 \%$ (nicotine $n=7$, saline $n=6$ ). Representative blots comparing saline (S) vs nicotine $(\mathrm{N})$-treated animals are shown for each subunit. *Significantly different from saline; $p<0.05$, unpaired $t$-test.

VTA. These findings demonstrate that chronic nicotine SA selectively modulates ionotropic glutamate receptor subunit expression levels, suggesting that glutamatergic neurotransmission is altered within the mesocorticolimbic pathway.

One study determined the response of ionotropic glutamate receptor mRNA levels, but not protein subunits, to chronic treatment with nicotine in vivo. It indicated 


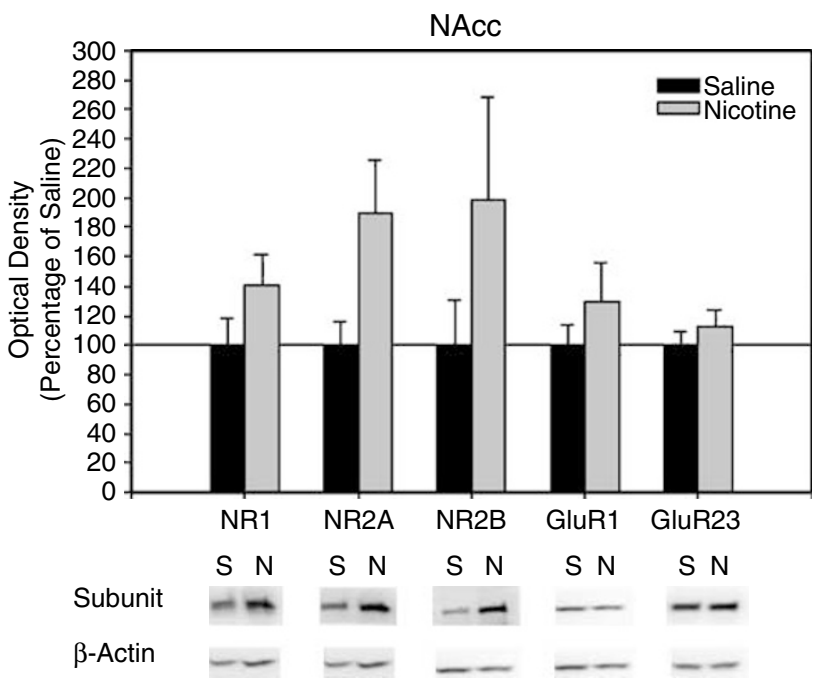

Figure 4 Nicotine SA ( 8 days) produced no statistically significant alterations in any of the subunits within the nucleus accumbens (NAcc) shell of rat brain (nicotine $n=7$, saline $n=6$ ). Representative blots comparing saline $(\mathrm{S})$ vs nicotine $(\mathrm{N})$-treated animals are shown for each subunit.

ST

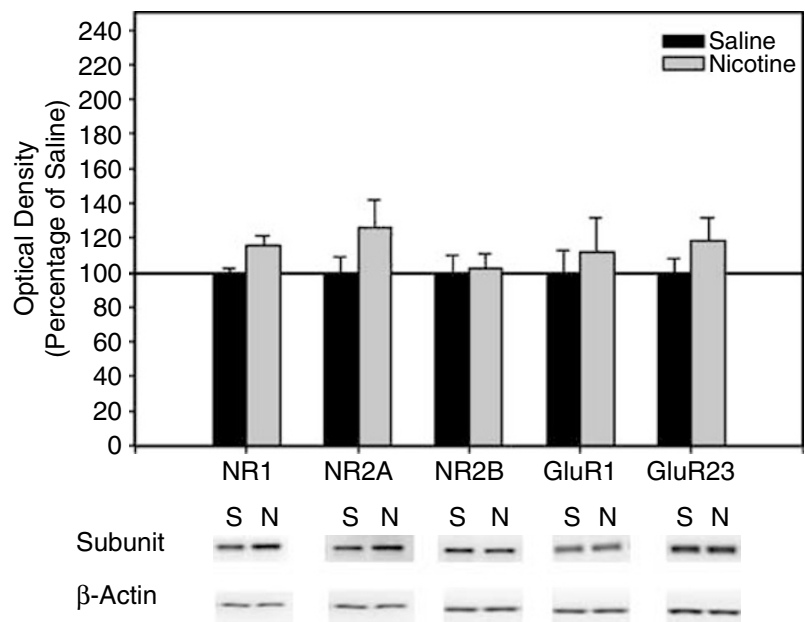

Figure 5 Nicotine SA ( 8 days) produced no statistically significant alterations in any of the subunits within the dorsal striatum (ST) of rat brain (nicotine $n=7$, saline $n=6$ ). Representative blots comparing saline (S) vs nicotine $(\mathrm{N})$-treated animals are shown for each subunit.

that chronic nicotine exposure during postnatal days 8-12 significantly increased NR2A mRNA levels in auditory cortex, whereas thalamic NR2B mRNA levels declined (Hsieh et al, 2002). This finding suggests that chronic exposure to nicotine affects the transcription of specific ionotropic glutamate receptor subunits in certain brain regions. However, subunit expression was only studied during the period of rat postnatal brain maturation equivalent to the third trimester of human brain development.

Several drugs of abuse have been shown to impact the function of ionotropic glutamate receptors. A recent study suggested that glutamatergic neurotransmission was altered after a single exposure to several drugs of abuse (Saal et al, 2003). In that study, $24-30 \mathrm{~h}$ after in vivo exposure (i.p. injection) to nicotine, morphine, ethanol, cocaine, or amphetamine, the strength of excitatory synapses on dopamine neurons was enhanced, reflecting an increase of AMPA/NMDA EPSC (Saal et al, 2003). However, the molecular mechanism(s) underlying these synaptic effects on midbrain dopamine neurons was not identified. The time interval between drug exposure and the detection of increased synaptic strength was probably sufficient for increased ionotropic glutamate subunit expression to have occurred.

One may postulate that chronic exposure to abused drugs can induce an increase in AMPA receptor subunit expression. This is supported by experiments showing that chronic cocaine treatment upregulated GluR1 and NR1 subunit levels in VTA, and that escalating doses of morphine also enhanced the expression of GluR1 subunits in VTA (Fitzgerald et al, 1996). In addition, after 3 days of withdrawal from repeated administration of cocaine or amphetamine, the direct application of AMPA onto VTA dopamine neurons resulted in increased glutamate responsiveness of these neurons (Zhang et al, 1997). Consistent with these findings, in the current study, chronic nicotine SA increased GluR2/3 subunit levels by $30 \%$ in VTA. This finding suggests that there might be a larger pool of recycling GluR2/3 subunits in VTA neurons since these subunits are known to recycle constitutively, which is important for the maintenance of basal synaptic activity (Passafaro et al, 2001; Shi et al, 2001). We also speculate that the AMPA/NMDA EPSC ratio may be increased if more GluR2/3 subunits reside in the plasma membrane. Interestingly, immunocytochemical studies have shown that GluR2/ 3 subunits were found in $63 \%$ of tyrosine hydroxylase (TH)positive VTA neurons, whereas GluR1 subunits were in $20 \%$ (Chen et al, 2001). Thus, the upregulated GluR2/3 subunits induced by chronic nicotine SA might be present in 'silent synapses', which had no AMPA receptors prior to nicotine SA, and in the GluR1/TH double-positive neurons, as well as GABAergic interneurons within VTA.

All drugs of abuse appear to enhance dopamine secretion in the NAcc (Berridge and Robinson, 1998). Nicotine does so in part through presynaptic $\alpha 7$ subunit-containing nicotinic receptors, located on glutamatergic terminals, which synapse on dopaminergic neurons within VTA (Mansvelder and McGehee, 2000). Nicotine stimulates glutamate release, increasing dopaminergic neurotransmission (Mansvelder and McGehee, 2000). Since the primary excitatory inputs to VTA originate in the PFC (Picciotto and Corrigall, 2002), enhanced excitatory input based on PFCdependent mechanisms also may be a factor. We found that PFC NR2A and NR2B subunit levels were upregulated by approximately 67 and $83 \%$, respectively, after nicotine SA. As the dendrites and soma of PFC glutamatergic neurons, which project to VTA, themselves receive glutamatergic inputs (Steketee, 2003), it is conceivable that the increased expression of PFC NMDA receptors containing NR2A and NR2B enhance the excitatory glutamatergic input to VTA. Similarly, a previous study found that chronic ethanol exposure increased NMDA (ie NR1, NR2A, and NR2B) and AMPA (ie GluR2/3) receptor subunit levels in primary cortical cultures, suggesting that increased activity of PFC glutamatergic neurons may be a mechanism common to multiple drugs of abuse (Chandler et al, 1999). 
Previous studies indicate that availability of NR2 subunits may be a limiting variable in the expression of intact NMDA receptors on the plasma membrane, as about $50 \%$ of NR1 is located in cytoplasmic pools while approximately $90 \%$ of NR2B is located at the cell surface (Hall and Soderling, 1997). Although the NR2B subunit appears to be constitutively trafficked to the synapse, NR2A overexpression leads to its synaptic incorporation in an activity-dependent manner (Barria and Malinow, 2002). Collectively, these observations suggest that the nicotine SA-induced increase in PFC NR2A and $2 \mathrm{~B}$ are likely to be incorporated in functional NMDA receptors.

Compared with VTA, NAcc may be less sensitive to the excitatory effects of glutamate, as evident in single cell recordings in vivo, made on the fifth day of treatment with cocaine or amphetamine (White et al, 1995). The overall current response of VTA dopamine neurons to glutamate was significantly elevated in amphetamine- and cocaine-treated rats, whereas this was not observed in NAcc neurons (White et al, 1995). Consistent with this, we did not observe significant changes in the expression of either NMDA or AMPA receptor subunits in the dopamine terminal regions (eg NAcc and ST) of animals chronically self-administering nicotine. This indicates the regional specificity of the changes in glutamate receptor subunit expression induced by chronic nicotine SA. Although these regional changes in glutamate receptor subunit expression may impact neuronal function, perhaps underlying alterations in the glutamatergic responsiveness of VTA dopamine neurons exposed to drugs of abuse, additional studies beyond Western immunoblotting are necessary to elucidate the implications of the present findings. Furthermore, other adaptive changes (eg posttranslational changes such as phosphorylation) may occur in some regions where subunit protein levels were unaffected (White et al, 1995).

To our knowledge, this is the first report of altered ionotropic glutamate receptor subunit expression during chronic nicotine SA. Based on these findings, we hypothesize that there exists a hyperglutamatergic state within the mesocorticolimbic pathway during chronic nicotine SA (Kalivas, 2004). This may be involved in aspects of drug SA, such as sensitization, that reflect the occurrence of increasing neuronal plasticity with duration of exposure (Wolf et al, 2004). Further investigation will be needed to evaluate the neurochemical changes underlying this hypothesis. In summary, chronic nicotine SA selectively upregulated NR2A and NR2B receptor subunits in PFC and GluR2/3 in VTA. These findings may implicate hyperglutamatergic neurotransmission within the mesocorticolimbic dopaminergic circuitry.

\section{ACKNOWLEDGEMENTS}

This study was supported by NIDA grant DA03977 to BMS. No commercial sponsorship was involved.

\section{REFERENCES}

Barria A, Malinow R (2002). Subunit-specific NMDA receptor trafficking to synapses. Neuron 35: 345-353.
Berridge KC, Robinson TE (1998). What is the role of dopamine in reward: hedonic impact, reward learning, or incentive salience? Brain Res Brain Res Rev 28: 309-369.

Chandler LJ, Norwood D, Sutton G (1999). Chronic ethanol upregulates NMDA and AMPA, but not kainate receptor subunit proteins in rat primary cortical cultures. Alcohol Clin Exp Res 23: 363-370.

Chen LW, Wei LC, Lang B, Ju G, Chan YS (2001). Differential expression of AMPA receptor subunits in dopamine neurons of the rat brain: a double immunocytochemical study. Neuroscience 106: 149-160.

Corrigall WA, Franklin KB, Coen KM, Clarke PB (1992). The mesolimbic dopaminergic system is implicated in the reinforcing effects of nicotine. Psychopharmacology (Berl) 107: 285-289.

Ferrari R, Le Novere N, Picciotto MR, Changeux JP, Zoli M (2002). Acute and long-term changes in the mesolimbic dopamine pathway after systemic or local single nicotine injections. Eur J Neurosci 15: 1810-1818.

Fitzgerald LW, Ortiz J, Hamedani AG, Nestler EJ (1996). Drugs of abuse and stress increase the expression of GluR1 and NMDAR1 glutamate receptor subunits in the rat ventral tegmental area: common adaptations among cross-sensitizing agents. J Neurosci 16: 274-282.

Fu Y, Matta SG, Gao W, Brower VG, Sharp BM (2000). Systemic nicotine stimulates dopamine release in nucleus accumbens: re-evaluation of the role of $N$-methyl-D-aspartate receptors in the ventral tegmental area. J Pharmacol Exp Ther 294: 458-465.

Hall RA, Soderling TR (1997). Differential surface expression and phosphorylation of $N$-methyl-D-aspartate receptor subunits NR1 and NR2 in cultured hippocampal neurons. J Biol Chem 272: 4135-4140.

Hsieh CY, Chen Y, Leslie FM, Metherate R (2002). Nicotine exposure during a postnatal critical period alters NR2A and NR2B mRNA expression in rat auditory forebrain. Brain Res Dev Brain Res 133: 19-25.

Kalivas PW (2004). Recent understanding in the mechanisms of addiction. Curr Psychiatry Rep 6: 347-351.

Le Moal M, Simon H (1991). Mesocorticolimbic dopaminergic network: functional and regulatory roles. Physiol Rev 71: 155-234.

Mansvelder HD, McGehee DS (2000). Long-term potentiation of excitatory inputs to brain reward areas by nicotine. Neuron 27: 349-357.

Nakanishi S, Nakajima Y, Masu M, Ueda Y, Nakahara K, Watanabe $\mathrm{D}$ et al (1998). Glutamate receptors: brain function and signal transduction. Brain Res Brain Res Rev 26: 230-235.

Passafaro M, Piech V, Sheng M (2001). Subunit-specific temporal and spatial patterns of AMPA receptor exocytosis in hippocampal neurons. Nat Neurosci 4: 917-926.

Paxinos G, Watson C (1986). The Rat Brain in Stereotaxic Coordinates, 2nd edn. Academic Press: New York.

Picciotto MR, Corrigall WA (2002). Neuronal systems underlying behaviors related to nicotine addiction: neural circuits and molecular genetics. J Neurosci 22: 3338-3341.

Risso F, Parodi M, Grilli M, Molfino F, Raiteri M, Marchi M (2004). Chronic nicotine causes functional upregulation of ionotropic glutamate receptors mediating hippocampal noradrenaline and striatal dopamine release. Neurochem Int 44: 293-301.

Saal D, Dong Y, Bonci A, Malenka RC (2003). Drugs of abuse and stress trigger a common synaptic adaptation in dopamine neurons. Neuron 37: 577-582.

Sesack SR, Pickel VM (1992). Prefrontal cortical efferents in the rat synapse on unlabeled neuronal targets of catecholamine terminals in the nucleus accumbens septi and on dopamine neurons in the ventral tegmental area. J Comp Neurol 320: 145-160.

Shi S, Hayashi Y, Esteban JA, Malinow R (2001). Subunit-specific rules governing AMPA receptor trafficking to synapses in hippocampal pyramidal neurons. Cell 105: 331-343. 
Steketee JD (2003). Neurotransmitter systems of the medial prefrontal cortex: potential role in sensitization to psychostimulants. Brain Res Brain Res Rev 41: 203-228.

Taber MT, Das S, Fibiger HC (1995). Cortical regulation of subcortical dopamine release: mediation via the ventral tegmental area. J Neurochem 65: 1407-1410.

Valentine JD, Hokanson JS, Matta SG, Sharp BM (1997). Acquisition of self-administration in rats allowed unlimited access to nicotine. Psychopharmacology 133: 300-304.

Watkins SS, Koob GF, Markou A (2000). Neural mechanisms underlying nicotine addiction: acute positive reinforcement and withdrawal. Nicotine Tob Res 2: 19-37.
White FJ, Hu XT, Zhang XF, Wolf ME (1995). Repeated administration of cocaine or amphetamine alters neuronal responses to glutamate in the mesoaccumbens dopamine system. J Pharmacol Exp Ther 273: 445-454.

Wolf ME, Sun X, Mangiavacchi S, Chao SZ (2004). Psychomotor stimulants and neuronal plasticity. Neuropharmacology 47(Suppl 1): 61-79.

Zhang XF, Hu XT, White FJ, Wolf ME (1997). Increased responsiveness of ventral tegmental area dopamine neurons to glutamate after repeated administration of cocaine or amphetamine is transient and selectively involves AMPA receptors. J Pharmacol Exp Ther 281: 699-706. 